\section{Bloody signals: From birth to disease and death}

The 2014 International Society for Experimental Hematology (ISEH) meeting in Montreal was filled with highimpact science spanning development, aging, immunity, cell fate, and leukemia, as well as the role of the niche in each of these processes. There were old friends and new faces filling the meeting rooms and dance floors at the conference. Meeting organizers led by ISEH presidentelect Paul Frenette and president Margaret Goodell brought together leaders in the field as well as newcomers to present their most recent and compelling work, which we summarize below.

\section{Stem cell niche}

Although the $43^{\text {rd }}$ annual ISEH scientific meeting was not a stem cell niche-specific conference, it gathered some of the top researchers in the field. The recipients of the prestigious ISEH awards were Toshio Suda (Donald Metcalf Award) and Emmanuelle Passegué (Mc Culloch and Till Award), both of whom have made major contributions to the field of the stem cell niche.

Professor Suda (Keio University, Tokyo, Japan) has contributed to the elucidation of the stem cell niche and establishing the fields of stem cell metabolism and stem cell aging $[1,2]$. He developed a single-cell culture system and found that hematopoietic stem cells (HSCs) use the glycolytic pathway to reduce oxidative stress. Regarding aging, he presented data showing that a protein called POT1 prevents telomere instability; however, its expression is reduced with age.

Vascular signals are important in the HSC niche. Diana Ramirez-Bergeron (Case Cardiovascular Research Institute, Cleveland, $\mathrm{OH}$ ) provided evidence that the transcriptional activity of HIF in the endothelium is important to maintain the vascular niche and respond to stress. Myriam Grunwald (Haddassah Medical School, Jerusalem, Israel) showed that ectopic overexpression of VEGF in the liver is sufficient to establish an active ectopic hematopoietic niche. Ingmar Bruns (Albert Einstein College of Medicine, New York, NY) showed that, in addition to the vascular niche,

Offprint requests to: Dr. Carolina Abramovich; E-mail: exphem@telus.net megakaryocytes adjacent to blood vessels support HSC quiescence via CXCL4.

The importance of the niche on hematologic disease was discussed. Simón Mendez-Ferrer gave the New Investigator's Lecture, where he presented work from his group demonstrating alterations to both HSCs and niche in myeloproliferative neoplasms (MPNs), challenging the paradigm of MPN driven solely by mutated HSCs [3]. Andreas Trumpp (German Cancer Research Center, Heidelberg, Germany) and Andreas Reinisch (Stanford University, Stanford, CA) presented different methods to "humanize" mouse models to improve engraftment of human hematopoietic stem and progenitor cells (HSPCs) from patients with myelodysplastic syndrome (MDS) or primary myelofibrosis, respectively [4]. Both groups demonstrated that engraftment of patient-derived mesenchymal stromal cells improved the in vivo growth of diseased HSPCs, opening the doors to understanding niche-HSPC connections in these difficult-to-model diseases.

To gain insight into niche regulation of HSCs, Linheng Li (Stowers Institute, Kansas City, MO), presented a transcriptional approach to survey gene expression in various niche and HSPC compartments. His findings suggest that different niche locations provide unique sites that maintain and retain different HSC states, which supports his hypothesis of different HSC populations or pools within the bone marrow. Recently, Pierre Charbord and Charles Durand took a systems approach to define the signals from stromal cells that support or inhibit hematopoiesis [5]. Exploiting the ability to prospectively isolate phenotypically-defined adult HSPC populations, Andreas Trumpp and team (including Daniel Lipka, the 2014 postdoctoral prize winner) used high throughput transcriptomics, methylomics, and proteomics to generate a comprehensive map of molecular alterations occurring during fate transitions at the earliest steps of murine hematopoietic differentiation [6].

Developmental niches and the pertinent signals for HSC derivation and expansion were also discussed. Jalal Ahmed (Albert Einstein College of Medicine) studied the role of Nestin + pericytes in fetal liver and proved that these cells are the gatekeepers of HSC expansion in the fetal liver.

\section{Development}

During their inception, HSCs arise from specialized hemogenic endothelial cells and are controlled by specific cues. 
Roger Patient (Weatherall Institute of Molecular Medicine, Oxford University, Oxford, UK) led the way by discussing the importance of extrinsically derived BMP, FGF, and VEGF on hemogenic endothelium formation [7-9]. Elaine Dzierzak (University of Edinburgh, Edinburgh, UK) used highly sensitive RNA-seq to delineate the genes differentially expressed among aortic HSCs, hemogenic endothelial cells, and nonhemogenic endothelial cells, identifying GPR56 as a required receptor for hematopoietic cluster formation. Ann Zovein (University of California, San Francisco, CA) used a murine model to demonstrate that the timing of expression of Sox 17 and Notch1 is important for hematopoietic specification because early deletion prevents EHT, whereas later deletion promotes it. Albert Kim (University of California, San Diego, CA; 2014 student award winner) explored autonomous and nonautonomous roles for Notch receptors on HSC formation in a zebrafish model [10]. Mauricio Cortes (Beth Israel Deaconess Medical Center, Boston, MA) showed that vitamin D3 plays a dynamic role in HSC formation. The inactive form of vitamin $\mathrm{D}$ affects the vascular niche through downregulation of hedgehog signaling, whereas the active form increases HSC numbers both in zebrafish and in human CD $34^{+}$umbilical cord blood cells. David Traver (University of California, San Diego, CA) showed that the inflammatory signal $\mathrm{TNF} \alpha$ also plays a role in HSC formation through downregulation of Notch and nuclear factor kB. Alaa Arraf (Technicon Israel Institute of Technology, Haifa, Israel) studied the origins of aortic endothelial cells in the chick and found that coelomic epithelial-derived cells are incorporated in the ventral wall of the dorsal aorta and differentiate into CD45-expressing hematopoietic cells, suggesting a nonvascular origin for HSCs. Along a similar vein, Peter Currie's group recently published their data demonstrating a replacement of aortic endothelium with cells derived from the somite that provide a regulatory niche [11]. These reports suggest that there is still much to learn about the early origins of HSCs.

The mammalian embryo can also support the autonomous generation of other hematopoietic lineages before the emergence of HSCs. Kathleen McGrath (University of Rochester, Rochester, NY) demonstrated that yolk sacderived $\mathrm{Mac}^{+} \mathrm{Grl}^{+}$cells circulate from E11.5 and seed the fetal liver, showing the existence of neutrophilic populations before the emergence of HSCs. Jim Palis (University of Rochester) demonstrated that lymphoid cells can arise from the yolk sac independently of erythroidmyeloid progenitors and showed that an embryo needs not HSCs, but only erythroid-myeloid progenitors, to survive until birth. Sten Eirik Jacobsen (University of Oxford, Oxford, UK) also presented data on the emergence of lymphoid cells before the emergence of definitive HSCs [12]. Anna Beaudin (University of California, San Francisco) showed that there is a developmentally restricted HSC population, primarily generating lymphoid cells, that can support adult hematopoietic reconstitution but is not normally present in adults. The emerging data in support of the earlier formation of populations once thought to only arise from HSCs, as well as data demonstrating lineagebiased HSCs in development, call into question the dogmatic concept of a strict hierarchical structure of hematopoiesis [13].

Leaders in the field of pluripotent stem cells and reprogramming presented their most recent work on the generation of HSCs. Gordon Keller (University of Toronto, Toronto, Canada) discussed the need to identify and eliminate primitive hematopoietic cells from cultures to reveal and enrich definitive blood cells $[14,15]$. For example, retinoic acid and Wnt activators, as well as Activin/Nodal signaling inhibitors, selectively support the growth of definitive hematopoietic progenitors over primitive counterparts $[16,17]$. Pedro Real (GENyO, Granada, Spain) showed that expression of RUNX1c in human embryonic stem cells is critical for production of hematopoietic progenitors [18]. Derrick Rossi (Harvard Medical School, Boston, MA) and George Daley (Harvard Medical School) discussed strategies to dedifferentiate blood cells toward an HSClike state. Derrick Rossi's group demonstrated the ability of only six transcription factors to reprogram mature murine blood cells to functional long term HSCs [19]. Daley's group, including Linda Vo, who also presented at the conference, took a similar approach to redirect committed myeloid progenitors, using Sox4, Myb, ERG, HoxA9, and RORA to confer self-renewal and engraftability [20]. Surprisingly, there was no overlap in the factors used in the studies of Rossi and Daley, suggesting that there is more than one way to confer stemness. Both Rossi and Daley presented unpublished data about chemical screening approaches to make HSC out of non-HSCs.

Some scientists are using development to gain insight into the mechanisms of hematologic disease. Teresa Bowman (Albert Einstein College of Medicine) presented data showing that zebrafish with mutated $s f 3 b l$, a spliceosomal gene that is often mutated in MDS patients, has diminished HSC number but also increased DNA damage and p53 pathway activation. Troy Lund (University of Minnesota, Minneapolis, MN) presented a model to study the effects of oxidative stress on erythroid cells and showed that tp53 can act as an antioxidant critical for the erythroid response to pro-oxidants such as napthol [21].

\section{Stem cell fate}

Stem cell identity and the instruction of fate specification are known to be controlled in a precise manner through transcriptional and epigenetic regulatory circuits, yet many of the mechanisms are poorly understood.

Two presentations shed light on the mostly unknown mechanisms of action by which presumably globallyacting chromatin-remodeling enzymes exert their genespecific regulatory function during HSC fate determination. 
Sonia Cellot (University of Montreal, Montreal, Canada) showed that two Jumonji domain-containing histone demethylases have highly specific and opposing functions in HSCs, which could be exploited for HSC expansion (stimulating positive regulator Jhdm1f) and leukemia stem cell-directed therapies (enhancing the activity of negative regulator Jarid1b) [22]. Julie Lessard (University of Montreal, Montreal, Quebec, Canada) shared new data that hematopoietic SWI/SNF-like BAF complex composition is cell type and stage specific. In quiescent HSCs, the complex is assembled around the Brahma ATPase; upon entrance into cell cycle and in leukemic stem cells, Brg1 ATPase is utilized. Consistently, Brg1 is essential for leukemia maintenance but is dispensable for the maintenance of quiescent LT -HSCs [23].

Transcriptional and epigenetic regulation is also associated with the functional decline of many tissue-specific stem cells during aging and malignant transformation. Modeling hematologic myeloid malignancies in mice in adoptive bone marrow transplantation assays, Toshio Kitamura (University of Tokyo, Tokyo, Japan) identified new therapeutic targets for leukemia patients with c-terminally truncated ASXL1 [24]. Matilda Billing (Lund University, Lund, Sweden) showed new insights into the TGF $\beta$-mediated regulation of HSC quiescence, involving p57 and GATA2, which may also apply to other malignancies. SCL/ TAL1, which has a central role in T-ALL, was shown to confer oncogenic activity by inducing self-renewal capacity in a subset of double-negative thymocytes, thereby converting them into preleukemic stem cells, as presented by Trang Hoang (University of Montreal).

\section{Stem cell expansion}

Currently, bone marrow, peripheral blood, and cord blood represent the main sources of hematopoietic stem and progenitor cells used clinically to treat a variety of hematologic diseases. Due to the noninvasive isolation and lower immune matching requirements of cord blood cells, they are gaining popularity for transplantation, but many banked cords have insufficient cell numbers. Guy Sauvageau's group (University of Montreal) used high throughput chemical screening to identify UM171 as a potent small molecule that expands long term repopulating HSPCs [25]. Jonas Larsson's group (Lund University) screened for negative regulators of human HSPC expansion and demonstrated that diminished cohesins led to HSPC expansion skewed along the myeloid lineage [26]. These data are consistent with the suggested role of cohesin mutations in leukemia and demonstrate the value of in vivo validation of any expansion approaches to ensure no harm.

\section{Leukemia}

In 1994, the group of John E. Dick in Toronto provided the first compelling evidence that only a rare population of human acute myeloid leukemia cells display leukemiainitiating properties, whereas the bulk of the tumor does not show significant leukemogenic potential [27]. Two decades later, an impressive wealth of information has been gained on the existence of cancer stem cells in other solid malignancies, but numerous basic questions remain unanswered. Sean Morrison (Howard Hughes Medical Institute, Chicago, IL) and Scott Armstrong (Boston Children's Hospital, Boston, MA) provided a critical assessment of the progress thus far made in the understanding of the biology of cancer stem cells. Morrison argued that, although many acute and chronic myeloid leukemias follow a cancer stem cell (CSC) model, some solid tumors, such as melanoma, are uniformly tumorigenic under permissive conditions, which suggests that cell fate can be viewed as how environment shapes cellular potential [28]. Armstrong agreed with the sentiment that not all tumor types follow the cancer stem cell model, but showed compelling evidence in CML that differences between CSCs and bulk tumor could have important therapeutic implications [29]. Since it is still unclear how generalizable the cancer stem cell model is, we need to further address the role of CSCs in cancer biology.

Transcription factor dysregulation can be a major component of cancer initiation. Claus Nerlov (University of Edinburgh) identified a common molecular signature of leukemic stem cells transformed by diverse oncogenes from common myeloid progenitors. Tarik Möröy (IRCM, Montreal, Quebec, Canada) showed that the zinc finger protein Gfil maintains lymphoma development and progression by blocking the activation of the K372 monomethylated p53 [30]. Magali Humbert (University of Montreal) showed that LMO2 overexpression specifically influences cell cycle of thymic progenitors, which are targets of cell transformation in T-ALL and regulates DNA replication through direct binding (with other replication proteins) to known origins of replication in hematopoietic progenitors. Céline Moison (University of Montreal) demonstrated that E4f1, a binding partner of Tp53, Rb1, and Bmi1, is a critical regulator of the DNA damage response via interactions with Chk1. Transcription factors can also alter metabolism, leading to changes in cell state and tumorigenicity. Patricia Ernst (Dartmouth Medical School, Hanover, NH) presented the surprising finding that the methyltransferase activity of MLL is not required for its function in hematopoiesis or leukemia [31]. Yoshihiro Hayashi (Cincinnati Children's Hospital, Cincinnati, OH) determined that MDS- or acute myeloid leukemia (AML)-associated RUNX1 mutations act as cooperating oncogenes with MLL-PTD and showed that those cells are uniquely sensitive to inhibition of HIF- $1 \alpha$, which may guide therapeutic interventions for MDS. Keisuke Ito (Albert Einstein College of Medicine) described his work showing PML-PPAR $\delta$-fatty acid oxidation pathway controls asymmetric stem cell division and commitment [32]. Benjamin Ebert (Harvard Stem Cell Institute, Boston, 
MA) described a novel mechanism of therapeutic activity for lenalidomide reliant on direct binding to the CRBNDDB1 E3 ubiquitin ligase. Lenalidomide's selective growth inhibition in multiple myeloma (and likely other B-cell lymphomas) is mediated by targeted degradation of the IKZF1 and IZKF3 lymphoid transcription factors that are essential for the differentiation and survival of plasma cells [33]. These findings identify the multifaceted role of transcription factors in manipulation of leukemia stem cell function, with potential therapeutic implications.

The modern era of cancer genomics was well covered at this meeting, with talks from leaders in the field. As part of Margaret Goodell's Presidential Symposium highlighting use of single-cell technologies, Anthony Green (University of Cambridge, Cambridge, UK) identified somatic mutations in the endoplasmic reticulum chaperone CALR in $70 \%-84 \%$ of patients with MPNs lacking JAK2 mutations [34]. He demonstrated that gene dosage (JAK2V617 F) and order of mutation (TET2 $\rightarrow$ JAK2 vs. JAK2 $\rightarrow$ TET2) influence disease progression [35]. Timothy Ley (Washington University Medical School, St. Louis, MO) presented whole genome sequencing data from 200 AML patients and identified many important rules of interaction or mutual exclusion among recurrently mutated genes. He also showed that many clones were only detectable after treatment. Ravi Majeti (Stanford University) presented a genomic analysis of highly purified leukemic and residual normal HSCs from de novo AML patients that supports the concept of a reservoir of preleukemic HSCs harboring founder mutations lacking the complete complement of abnormalities required to generate AML [36,37]. Lambert Busque (Hôpital Maisonneuve-Rosemont, Montreal, Quebec, Canada) provided evidence that mutations in genes associated with hematologic cancers, such as TET2, can occur in aging subjects and may be the elusive early events in the pathogenesis of these disorders [38]. Connie Eaves (BC Cancer Agency, Vancouver, British Columbia, Canada) described her newest work using DNA barcodes to assess clonal architecture, revealing an even greater diversity than previously anticipated in the size, content, durability and reversible dormancy of clones produced by normal hematopoietic and mammary cells and their transformed derivatives. In-depth clinical sequencing is required to identify the genes that are mutated in each sample, but also to define clonal architecture and how subclones individually respond to induction therapy.

\section{Inflammation and immunity}

Since the seminal discoveries by Andreas Trumpp and Margaret Goodell's labs about the effects of interferon (IFN) $\alpha$ and IFN $\gamma$, respectively, on HSCs, many groups are studying the role of inflammatory signaling pathways in HSCs $[39,40]$. Katherine King (Baylor College of Medicine, Houston, TX) showed that myeloid-based HSCs express higher levels of IFN $\gamma$ and enter cell cycle after stimulation with IFN $\gamma$ or infection, thus providing a novel way to discriminate alternate HSC phenotypes. Simon Haas (DKFZ) showed that an interplay between noncanonical type I interferon and mTOR pathways activates megakaryocyte transcripts and primes HSCs to megakaryocytic differentiation. Brian Liddicoat (St Vincent's Institute, Fitzroy, Victoria, Australia) provided interesting results that a catalytically inactive mutant of ADAR1, which modulates IF$\mathrm{N} \alpha$ responses, also leads to failed hematopoiesis that can be rescued by knocking out MDA5. Regulation of IFN $\gamma$ responses is also important. Fanching Lin (National Cancer Institute, Frederick, MD) showed that generation of CMP, GMP and MEP from multipotent progenitors is inhibited in mice that overexpress IFN $\gamma$.

Another cytokine, M-CSF, is responsible for myeloid priming of HSCs under stress conditions. Sandrine Sarrazin (Centre d'Immunologie de Marseille-Luminy, Marseille, France) showed that the transcription factor MafB is the gatekeeper of the myeloid cell fate in HSCs by regulating the accumulation of Pu.1 [41]. Hao Yuan Kueh (California Institute of Technology, Pasadena, CA), experimenting with macrophage and T-cell fate control, showed that developing macrophages lengthen their cell cycle to promote PU.1 expression, whereas T-cells use Notch signaling to switch $\mathrm{Bcl11} \mathrm{b}$ from an inactive to an active state and thus regulate cell fate [42].

Another major topic was the role of immune cells in disease. Josée Hébert (Centre hospitalier de l'Université de Montréal, Montreal, Quebec, Canada) elegantly reviewed how extracellular vesicles can modulate the humoral autoimmune response and control solid organ transplants and graft-versus-host disease (GVHD). Toward modulating GVHD, Marcel Van Der Brink (Memorial Sloan Kettering Cancer Center, New York, NY) reported on a regenerative loop in intestines, thymus, and possibly liver that relies on the production of interleukin 22 from tissue-specific dendritic cells and can alleviate epithelial cell damage. In addition, Denis Claude Roy (Hôpital Maisonneuve-Rosemont) showed that photosensitizer dibromorhodamine can be used to eliminate alloreactive T-cells and prevent GVHD [43]. T cells can also be exploited in cancer therapies. Michel Sadelain (Memorial Sloan Kettering Cancer Center) talked about the role of chimeric antigen receptor-expressing $\mathrm{T}$ cells and their effectiveness in multiple cancers, including CLL, NHL, and ALL [44-46]. In concert with this notion, Claude Perreault (University of Montreal) demonstrated the importance of the immunopeptidome for combating disease and explained how important it is to combine proteomic analysis with transcriptome data [47].

\section{Aging}

Aging leads to the generation of more phenotypically defined but less functionally competent HSCs. The session on aging explored why this occurs and ways in which aged 
HSCs might be rejuvenated. Hartmut Geiger (Cincinnati Children's Hospital; University of Ulm, Ulm, Germany) presented evidence regarding the role of cell polarity in HSC function. Young HSCs demonstrate both nuclear and cytoplasmic polarity with respect to proteins including Cdc42, tubulin, and AcH4K16 [48]. Geiger postulates that ageing may be attributed to loss of polarization of epigenetic marks, such as H4K16 acetylation. Christopher Park (Memorial Sloan Kettering Cancer Center) reported that miR125b maintains HSC self-renewal but that its level is diminished with age. Restoring expression of miR $125 \mathrm{~b}$ in aged HSCs could reverse age-related phenotypes. To discover the expression signature of aged HSCs, Monika Kowalczyk (Broad Institute, Cambridge, MA) reported studies using single-cell RNAseq of HSCs that revealed differences in cell cycle status. Larry Luchsinger (Columbia University) provided compelling data that identified a novel molecular switch instructing the differentiation of either myeloid or lymphoid lineage-primed HSCs through Prdm16-mediated regulation of mitochondrial dynamics and calcium homeostasis [49].

Themes of aging, calorie restriction, stress, and cell cycle regulation were all echoed in Emmanuelle Passegué's (University of California, San Francisco) lecture as the 2014 recipient of the McCulloch and Till Award. Among her many contributions to the field of HSC biology, Passegué's work has spanned the areas of metabolic and environmental stress, DNA damage repair, aging, and leukemogenesis [50-52]. Recent work in the Passegue lab indicates that the multipotent progenitor stage of hematopoietic development may be the critical stage at which lineage commitment first occurs. Transcriptional differences in these classes of multipotent progenitors fall into three major components: metabolism, cell cycle, and inflammation. Further insight into important stages in differentiation has come through recent in situ studies of malignant AML clusters showing that proliferation and differentiation are inversely correlated.

\section{Concluding remarks}

Many of this meeting's presentations demonstrated that dissecting the mechanisms that instruct cell fate decisions has become one of the leitmotifs, not only in our investigations of the fundamentals of normal hematopoiesis, but also in our quest for successful tissue engineering approaches for disease modeling and drug testing.

Eirini Trompouki

Cellular and Molecular Immunology, Max Planck Institute of Immunology and Epigenetics, Freiburg, Germany

Katherine Y. King Baylor College of Medicine, Houston, TX, USA

Britta Will Albert Einstein College of Medicine, Bronx, NY, USA
Julie Lessard IRIC, Université de Montréal, Montréal, Québec, Canada

Eugenia Flores-Figueroa Oncological Research Unit at the Mexican Institute of Social Health Mexico City, Mexico

Konstantinos D. Kokkaliaris ETH Zurich, Department of Biosystems Science and Engineering, Basel, Switzerland

Teresa Bowman Albert Einstein College of Medicine, Bronx, NY, USA

\section{References}

1. Ito K, Suda T. Metabolic requirements for the maintenance of selfrenewing stem cells. Nat Rev Mol Cell Biol. 2014;15:243-256.

2. Nakamura-Ishizu A, Suda T. Aging of the hematopoietic stem cells niche. Int J Hematol. 2014;100:317-325.

3. Arranz L, Sánchez-Aguilera A, Martín-Pérez D, et al. Neuropathy of haematopoietic stem cell niche is essential for myeloproliferative neoplasms. Nature. 2014;512:78-81.

4. Medyouf H, Mossner M, Jann JC, et al. Myelodysplastic cells in patients reprogram mesenchymal stromal cells to establish a transplantable stem cell niche disease unit. Cell Stem Cell. 2014;14: 824-837.

5. Charbord P, Pouget C, Binder H, et al. A systems biology approach for defining the molecular framework of the hematopoietic stem cell niche. Cell Stem Cell. 2014;15:376-391.

6. Cabezas-Wallscheid N, Klimmeck D, Hansson J, et al. Identification of regulatory networks in HSCs and their immediate progeny via integrated proteome, transcriptome, and DNA methylome analysis. Cell Stem Cell. 2014;15:507-522.

7. Ciau-Uitz A, Monteiro R, Kirmizitas A, Patient R. Developmental hematopoiesis: ontogeny, genetic programming and conservation. Exp Hematol. 2014;42:669-683.

8. Ciau-Uitz A, Pinheiro P, Gupta R, Enver T, Patient R. Tel1/ETV6 specifies blood stem cells through the agency of VEGF signaling. Dev Cell. 2010;18:569-578.

9. Wilkinson RN, Pouget C, Gering M, et al. Hedgehog and Bmp polarize hematopoietic stem cell emergence in the zebrafish dorsal aorta. Dev Cell. 2009;16:909-916.

10. Kim AD, Melick CH, Clements WK, et al. Discrete Notch signaling requirements in the specification of hematopoietic stem cells. EMBO J. 2014;33:2363-2373.

11. Nguyen PD, Hollway GE, Sonntag C, et al. Haematopoietic stem cell induction by somite-derived endothelial cells controlled by meox 1 . Nature. 2014:512:314-318.

12. Boiers C, Carrelha J, Lutteropp M, et al. Lymphomyeloid contribution of an immune-restricted progenitor emerging before definitive hematopoietic stem cells. Cell Stem Cell. 2013;13:535-548.

13. Frame JM, McGrath KE, Palis J. Erythro-myeloid progenitors: "definitive" hematopoiesis in the conceptus before the emergence of hematopoietic stem cells. Blood Cells Mol Dis. 2013; 51:220-225.

14. Kennedy M, Awong G, Sturgeon CM, et al. T lymphocyte potential marks the emergence of definitive hematopoietic progenitors in human pluripotent stem cell differentiation cultures. Cell Rep. 2012;2:1722-1735.

15. Clarke RL, Yzaguirre AD, Yashiro-Ohtani Y, et al. The expression of Sox 17 identifies and regulates haemogenic endothelium. Nat Cell Biol. 2013;15:502-510. 
16. Chanda B, Ditadi A, Iscove NN, Keller G. Retinoic acid signaling is essential for embryonic hematopoietic stem cell development. Cell. 2013; 155:215-227.

17. Sturgeon CM, Ditadi A, Awong G, Kennedy M, Keller G. Wnt signaling controls the specification of definitive and primitive hematopoiesis from human pluripotent stem cells. Nat Biotechnol. 2014; 32:554-561.

18. Real PJ, Navarro-Montero O, Ramos-Mejía V, Ayllón V, Bueno C, Menéndez P. The role of RUNX1 isoforms in hematopoietic commitment of human pluripotent stem cells. Blood. 2013;121:5250-5252.

19. Riddell J, Gazit R, Garrison BS, et al. Reprogramming committed murine blood cells to induced hematopoietic stem cells with defined factors. Cell. 2014;157:549-564.

20. Doulatov S, Vo LT, Chou SS, et al. Induction of multipotential hematopoietic progenitors from human pluripotent stem cells via respecification of lineage-restricted precursors. Cell Stem Cell. 2013;13: 459-470.

21. Patrinostro X, Carter ML, Kramer AC, Lund TC. A model of glucose-6-phosphate dehydrogenase deficiency in the zebrafish. Exp Hematol. 2013;41:697-710.

22. Cellot S, Hope KJ, Chagraoui J, et al. RNAi screen identifies Jarid $1 b$ as a major regulator of mouse HSC activity. Blood. 2013; 122:1545-1555.

23. Buscarlet M, Krasteva V, Ho L, et al. Essential role of BRG the ATPase subunit of BAF chromatin remodeling complexes, in leukemia maintenance. Blood. 2014;123:1720-1728.

24. Inoue D, Kitaura J, Togami K, et al. Myelodysplastic syndromes are induced by histone methylation-altering ASXL1 mutations. J Clin Invest. 2013;123:4627-4640.

25. Fares I, Chagraoui J, Gareau Y, et al. Cord blood expansion. Pyrimidoindole derivatives are agonists of human hematopoietic stem cell self-renewal. Science. 2014;345:1509-1512.

26. Karlsson C, Rak J, Larsson J. RNA interference screening to detect targetable molecules in hematopoietic stem cells. Curr Opin Hematol. 2014;21:283-288.

27. Lapidot T, Sirard C, Vormoor J, et al. A cell initiating human acute myeloid leukaemia after transplantation into SCID mice. Nature. 1994;367:645-648.

28. Quintana E, Piskounova E, Shackleton M, et al. Human melanoma metastasis in NSG mice correlates with clinical outcome in patients. Sci Transl Med. 2012;4:159ra149.

29. Heidel FH, Bullinger L, Feng Z, et al. Genetic and pharmacologic inhibition of beta-catenin targets imatinib-resistant leukemia stem cells in CML. Cell Stem Cell. 2012;10:412-424.

30. Khandanpour C, Phelan JD, Vassen L, et al. Growth factor independence 1 antagonizes a p53-induced DNA damage response pathway in lymphoblastic leukemia. Cancer Cell. 2013;23:200-214.

31. Mishra BP, Zaffuto KM, Artinger EL, et al. The histone methyltransferase activity of MLL1 is dispensable for hematopoiesis and leukemogenesis. Cell Rep. 2014;7:1239-1247.

32. Ito K, Carracedo A, Weiss D, et al. A PML-PPAR- $\delta$ pathway for fatty acid oxidation regulates hematopoietic stem cell maintenance. Nat Med. 2012;18:1350-1358.

33. Kronke J, Udeshi ND, Narla A, et al. Lenalidomide causes selective degradation of IKZF1 and IKZF3 in multiple myeloma cells. Science. 2014;343:301-305.
34. Nangalia J, Massie CE, Baxter EJ, et al. Somatic CALR mutations in myeloproliferative neoplasms with nonmutated JAK2. N Eng1 J Med. 2013;369:2391-2405.

35. Li J, Kent DG, Godfrey AL, et al. JAK2V617F homozygosity drives a phenotypic switch in myeloproliferative neoplasms, but is insufficient to sustain disease. Blood. 2014;123:3139-3151.

36. Corces-Zimmerman MR, Hong WJ, Weissman IL, Medeiros BC, Majeti R. Preleukemic mutations in human acute myeloid leukemia affect epigenetic regulators and persist in remission. Proc Natl Acad Sci U S A. 2014;111:2548-2553.

37. Jan M, Snyder TM, Corces-Zimmerman MR, et al. Clonal evolution of preleukemic hematopoietic stem cells precedes human acute myeloid leukemia. Sci Trans1 Med. 2012;4:149ra118.

38. Busque L, Patel JP, Figueroa ME, et al. Recurrent somatic TET2 mutations in normal elderly individuals with clonal hematopoiesis. Nat Genet. 2012;44:1179-1181.

39. Baldridge MT, King KY, Boles NC, Weksberg DC, Goodell MA Quiescent haematopoietic stem cells are activated by IFN-gamma in response to chronic infection. Nature. 2010;465:793-797.

40. Essers MA, Offner S, Blanco-Bose WE, et al. IFNalpha activates dormant haematopoietic stem cells in vivo. Nature. 2009;458:904-908.

41. Sarrazin S, Mossadegh-Keller N, Fukao T, et al. MafB restricts MCSF-dependent myeloid commitment divisions of hematopoietic stem cells. Cell. 2009;138:300-313.

42. Kueh HY, Champhekar A, Nutt SL, Elowitz MB, Rothenberg EV. Positive feedback between PU.1 and the cell cycle controls myeloid differentiation. Science. 2013:341:670-673.

43. Bastien JP, Krosl G, Therien C, et al. Photodepletion differentially affects CD4+ Tregs versus CD4+ effector T cells from patients with chronic graft-versus-host disease. Blood. 2010;116:4859-4869.

44. Sadelain M. From the guest editor: The rise of CAR therapy: the CD19 paradigm, and beyond. Introduction. Cancer J. 2014;20:105-106.

45. Davila ML, Riviere I, Wang X, et al. Efficacy and toxicity management of 19-28z CAR T cell therapy in B cell acute lymphoblastic leukemia. Sci Transl Med. 2014;6:224ra225.

46. Davila ML, Kloss CC, Gunset G, Sadelain M. CD19 CAR-targeted T cells induce long-term remission and B Cell Aplasia in an immunocompetent mouse model of B cell acute lymphoblastic leukemia. PLoS One. 2013;8:e61338.

47. de Verteuil DA, Rouette A, Hardy MP, et al. Immunoproteasomes shape the transcriptome and regulate the function of dendritic cells. J Immunol. 2014;193:1121-1132.

48. Florian MC, Nattamai KJ, Dörr K, et al. A canonical to noncanonical Wnt signalling switch in haematopoietic stem-cell ageing. Nature 2013:503:392-396.

49. Aguilo F, Avagyan S, Labar A, et al. Prdm16 is a physiologic regulator of hematopoietic stem cells. Blood. 2011;117:5057-5066.

50. Flach J, Bakker ST, Mohrin M, et al. Replication stress is a potent driver of functional decline in ageing haematopoietic stem cells. Nature. 2014;512:198-202.

51. Mohrin M, Bourke E, Alexander D, et al. Hematopoietic stem cell quiescence promotes error-prone DNA repair and mutagenesis. Cell Stem Cell. 2010;7:174-185.

52. Warr MR, Binnewies M, Flach J, et al. FOXO3A directs a protective autophagy program in haematopoietic stem cells. Nature. 2013;494: $323-327$. 